\section{ORIGINAL RESEARCH}

S. Baldi

T. Zander

M. Rabellino

G. González

M. Maynar

\title{
Carotid Artery Stenting without Angioplasty and Cerebral Protection: A Single-Center Experience with up to 7 Years' Follow-Up
}

BACKGROUND AND PURPOSE: The use of cerebral protection during CAS in the treatment of carotid artery disease is matter of controversy. The purpose of this study was to evaluate the outcome of CASWBAP in a large cohort of patients, with $\leq 7$ years' follow-up.

MATERIALS AND METHODS: Two hundred thirty-six patients with 255 symptomatic carotid stenoses and/or with high-risk-morphology plaques of $>50 \%$ and asymptomatic plaques of $>70 \%$ were prospectively identified. Patients underwent neurologic and carotid US examination before the procedure and during follow-up at 1, 3, 6, and 12 months and annually thereafter. Plain films of the neck were obtained immediately after the procedure and then at 1 and 3 months.

RESULTS: Technical success was achieved in 253/255 (99\%) patients. Primary stent placement was successful in 248/253 (98\%) patients. Neurologic periprocedural complications within 30 days included $1(0.4 \%)$ nondisabling stroke, $1(0.4 \%)$ disabling stroke, 11 (4.3\%) TIAs, and $1(0.4 \%)$ death. The mean duration of follow-up was $23 \pm 1.4$ months (range, 3-84 months). During the follow-up period, there were 9 additional deaths ( 7 unrelated to the carotid disease and 2 stroke-related) and 2 strokes (in other vascular territories). The degree of stenosis decreased from a mean of $82 \%$ before the procedure to a mean of $30 \%$ immediately after. During follow-up, $38(14.8 \%)$ angioplasties were performed due to restenosis in $19(7.4 \%)$ patients, lack of stent expansion in $14(5.4 \%)$, or both in $5(1.9 \%)$.

CONCLUSIONS: CASWBAP is effective and safe with a low incidence of periprocedural complications, providing satisfactory long-term clinical results.

ABBREVIATIONS: CAS $=$ carotid artery stenting; CASWBAP $=$ carotid artery stenting without balloon angioplasty and protection; $\mathrm{CEA}=$ carotid endarterectomy; $\mathrm{CPD}=$ cerebral protection device; CTA = CT angiography; DWI = diffusion-weighted imaging; ICU = intensive care unit; MRA = MR angiography; NASCET = North American Symptomatic Carotid Endarterectomy Trial; $\mathrm{TCD}=$ transcranial Doppler sonography; TIA = transient ischemic attack; US = ultrasonography

$\mathbf{R}$ esults of most recent prospective randomized multicenter studies have failed to show a noninferiority of CAS compared with CEA in the treatment of patients with symptomatic carotid artery stenosis. ${ }^{1-3}$ However CAS is increasingly used as an alternative to endarterectomy and is widely used in both asymptomatic and symptomatic patients and even in young patients. $^{4-6}$

One limitation of CAS is the potential for embolic stroke caused by plaque dislodgement of atheromatous material during the procedure. In the past years, with the advent of CPDs, thromboembolic complications were significantly reduced. ${ }^{7,8}$ Nevertheless, their clinical efficacy and safety are still a matter of controversy because they increase the risk of arterial vasospasm, thrombosis, and dissection. ${ }^{9,10}$ Moreover, embolic signals have been observed by TCD during all phases of the carotid stent procedure, whereas in a recent study, more signals were detected with the use of filter devices. ${ }^{11}$

Therefore, it is rational to expect that less manipulation and fewer devices crossing the stenosis may reduce the risk of

Received August 2, 2010; accepted after revision August 31

From the Department of Endovascular Therapy, Hospiten Rambla Hospital, Santa Cruz de Tenerife, Spain.

Please address correspondence to Sebastián Baldi, MD, Department of Endovascular Therapy, Hospital Hospiten Rambla, Rambla General Franco 115, 38001 Santa Cruz de Tenerife, Spain; e-mail: sebaldi73@yahoo.com

DOI 10.3174/ajnr.A2375 stroke during CAS. Indeed, a preliminary experience in CASWBAP demonstrated a low 30 -day stroke/death rate. ${ }^{12}$

In this study, we report the short- and long-term results of 255 consecutive carotid artery stenoses treated with CASWBAP and evaluate its efficacy and safety in our experience.

\section{Materials and Methods}

\section{Patient Population}

We analyzed data regarding 236 patients with 255 arterial carotid stenoses who underwent CASWBAP between 2002 and 2009.

\section{Patient Selection}

Inclusion criteria were the following:

- Symptomatic patients with stenosis $>50 \%$

- Asymptomatic patients with stenosis $>70 \%$

- Asymptomatic patients with stenosis between 50\% and 70\% with Doppler US findings showing high-risk-morphology plaque and silent infarcts ipsilateral to the stenosis demonstrated by DWI or microembolism detected by TCD.

Exclusion criteria were the following:

- Previous stroke within 6 weeks before CAS

- Total-occlusion lesions

- Bleeding diathesis

- Cerebral vascular malformations, degenerative cerebral diseases, 
cerebral tumors, illness impeding informed consent, and life expectancy $<2$ years

- Previous CEA.

\section{Preprocedural Evaluation}

All patients underwent an examination by a neurologist prior to the procedure.

The indication for stent placement was given by Doppler US and digital subtraction angiography in most of the cases. In selected cases, CTA or MRA was also performed.

The morphology of plaque was assessed by US; the plaque morphology was classified into 4 grades $^{13-15}$ : Grades 1 and 2 were considered high-risk-morphology plaque, grade 3 was medium risk, and grade 4 was low risk of stroke. All patients with a high-risk-morphology plaque were treated if stenosis was $>50 \%$.

Digital subtraction angiography (Integris; Philips Healthcare, Best, the Netherlands) was performed before the endovascular intervention in all patients. The degree of stenosis before stent placement was quantified by using the NASCET criteria ${ }^{16}$ and ranged from $50 \%$ to $99 \%$, with a mean of $82 \%$.

Before treatment, baseline cerebral CT or MR imaging was performed in all patients except 13. Intraprocedural TCD was performed in 15 patients.

\section{Stent-Placement Procedure}

All patients gave informed consent and underwent the procedure according to a protocol approved by the institutional review board. Patients were pretreated with $75 \mathrm{mg}$ of clopidogrel and $100 \mathrm{mg}$ of aspirin at least 72 hours before treatment. Dual antiplatelet medication was maintained for at least 6 months after treatment, and aspirin, indefinitely. An intravenous bolus of 5000 IU of heparin was administered immediately after sheath placement.

All treatments were performed by neurointerventional radiologists (S.B., M.R., T.Z., and M.M.) with experience in CAS ranging between 6 and 20 years.

With the patient under local anesthesia, a femoral approach was used in 248 patients; in 6 patients, a direct carotid approach was used (for an extremely tortuous aortic arch in 4 or Leriche syndrome in 2); and in 1 patient, a brachial approach was performed.

In the patients in whom the access was the femoral artery, we used, in the first part of our experience, an $8 \mathrm{~F}$ guiding catheter mounted coaxially over a $6 \mathrm{~F}$ multipurpose catheter. Afterward we used a longsheath $(6 \mathrm{~F})$ technique to engage the common carotid artery. In both cases, the catheters were advanced into the common carotid artery and 0.035 -inch stiff support wires were placed in the external carotid artery.

Using a road-mapping technique, we crossed the stenotic lesion by using a 0.014 -inch guidewire. This was followed by advancement and deployment of a tapered stent (Acculink, Abbott, Santa Clara, California; or Protégé, ev3, Plymouth, Minnesota) of appropriate dimensions across the stenosis. Atropine was never administered prophylactically before stent deployment; it was used only if bradycardia occurred during the procedure.

In 5 patients in whom the stenosis (99\% tight stenosis) could not be passed with the stent-delivery system, predilation with a 2.5 - to 3.0-mm angioplasty balloon was performed before stent deployment.

A cerebral angiogram was obtained at the end of the procedure. Hemostasis of the puncture site was achieved with a percutaneous closure device (Angio-Seal; St Jude Medical, Minnetonka, Minnesota). After the procedure was completed, a plain film of the neck was

\begin{tabular}{lc}
\hline \multicolumn{2}{l}{ Table 1: Baseline characteristics of patients } \\
\hline Characteristic & No. \\
\hline Demography & 255 \\
Cases & 200 (186 patients) \\
Men & $55(50$ patients) \\
Women & 71 \\
Mean age (yr) & \\
Vascular risk factors & $141(55 \%)$ \\
Coronary artery disease & $181(70 \%)$ \\
Hypertension & $93(36 \%)$ \\
Diabetes mellitus & $123(48 \%)$ \\
Hyperlipidemia & $129(50 \%)$ \\
Smoker & $57(22 \%)$ \\
$>79$ years & $178(69 \%)$ \\
$>2$ risk factors & $102(40 \%)$ \\
$>3$ risk factors &
\end{tabular}

obtained in different oblique projections to document the expansion of the stent.

\section{Definitions and Follow-Up}

Postprocedural neurologic complications were defined as follows:

1) “TIA," any neurologic deficit that completely resolved within 24 hours

2) "Nondisabling," if symptoms lasted more $>7$ days but resolved within 30 days with no disability of functional significance (modified Rankin Scale score, $<3$ )

3) "Disabling," if the patient had a persistent neurologic deficit causing disability of functional significance for $>30$ days after the onset of the symptoms (modified Rankin Scale score, $>4$ )

4) "Fatal," if death occurred as a direct result of stroke at any time after the procedure.

Follow-up assessment was carried out, whenever possible, with CT or MR imaging (including DWI) within 48 hours after stent placement. On discharge, patients were followed up clinically and with Doppler US at 1, 3, 6, and 12 months and annually thereafter. In the first 100 patients, plain films of the neck were obtained immediately after the procedure and in the same time intervals for $\leq 12$ months; afterward, they were performed only at 1 and 3 months because we considered that further expansion of the stent was less likely to occur.

The statistical analysis was performed by means of descriptive calculations of percentages.

\section{Results}

The population study consisted of 236 patients, 50 women $(21 \%)$ and 186 men $(79 \%)$. The mean age was $71 \pm 9.18$ years (37-92 years), with 255 carotid artery stenoses. Of the treated arteries, 152 (59\%) were symptomatic and 103 (41\%) asymptomatic. Of the symptomatic group, 136 (89\%) had stenoses of $>70 \%$ and $16(11 \%)$ had $>50 \%$. Of the asymptomatic group, $93(90 \%)$ had stenoses of $>70 \%$ and $10(10 \%)$ had $>50 \%$ ( 8 presented with high-risk-morphology plaque, and 2, with microembolisms detected by TCD).

Vascular-risk-factor distribution is summarized in Table 1.

\section{Procedural Results}

Technical success was achieved in 253/255 (99\%). In 2 patients, it was not possible to cross the lesion due to tight calcified stenoses. Primary stent placement was successful in 248/ 


\begin{tabular}{lccc}
\hline \multicolumn{2}{c}{ Table 2: Degree of residual stenosis } \\
\hline Initial Stenosis & \multicolumn{3}{c}{ Immediate Results } \\
\cline { 2 - 4 }$\%$ Stenosis & $\%$ Stenosis & $\%$ & No. Cases \\
\hline \multirow{2}{*}{$82 \%(50-99 \%)$} & $30 \%(0 \%-70 \%)$ & $0 \%-30 \%$ & $156(62 \%)$ \\
& & $>50 \%$ & $79(31 \%)$ \\
\hline
\end{tabular}

$253(98 \%)$ patients. In $5(2 \%)$ patients, predilation with a small balloon was necessary before stent deployment.

The degree of stenosis decreased from a mean of $82 \%$ before the procedure to a mean of $30 \%$ immediately after stent placement. In 156 patients, the immediate residual stenosis was $<30 \%$; in 79 , between $30 \%$ and $50 \%$; and in 20 patients, $>50 \%$ (Table 2).

There were no TIAs or strokes during the 255 procedures. Seven (4\%) patients experienced intraprocedural bradycardia, and $2(1.1 \%)$, hypotension. None of the patients presented with asystole during the procedure. Four (2\%) patients developed a hyperperfusion syndrome consisting of headache and confusion but without CT evidence of hemorrhage.

MR imaging with DWI could be performed before and within 48 hours after the procedure in 82 patients; silent infarcts were detected in $7(8.5 \%)$.

\section{Thirty-Day Periprocedural Results}

During the 30-day periprocedural period, there were 11 $(4.3 \%)$ TIAs, all within the first 24 hours after stent placement, and $1(0.4 \%)$ disabling stroke, $1(0.4 \%)$ nondisabling stroke, and $1(0.4 \%)$ death ( 1 stent thrombosis at 11 days). Excluding TIAs, the total stroke/death rate was $1.2 \%$.

\section{Long-Term Follow-Up}

One hundred seventy-two patients could be followed up beyond the periprocedural period of 30 days, with a mean follow-up time of $23 \pm 1.4$ months (range, 3-84 months). During this period, there were 9 deaths $(5 \%, 2$ related to stroke [ 1 in the posterior circulation and 1 intracranial stent thrombosed at 36 days] and 7 not related to stroke post-cardiac surgery) and 3 strokes ( 2 nondisabling strokes in vascular territories different from the stented artery and 1 disabling ipsilateral stroke due to stent thrombosis in a patient in whom clopidogrel was suspended before cardiac surgery).

During follow-up, 38 (14.8\%) angioplasties were performed, due to restenosis in 19 (7.4\%) patients, lack of stent expansion in 14 patients $(5.4 \%)$, or both in $5(1.9 \%)$. We waited $\leq 3$ months for stent self-expansion; after this period, if the residual stenosis was $>50 \%$, angioplasty with cerebral protection was performed. In case of restenosis, angioplasty was performed when the stenosis was $>70 \%$. All patients who received secondary angioplasty were asymptomatic.

\section{Discussion}

In many centers, neuroprotected CAS has become the standard practice for the endovascular treatment of patients for whom surgery for symptomatic or asymptomatic severe carotid stenoses carries high risk. ${ }^{17-20}$ Some CAS trials in which CPDs were used demonstrated equivalence with endarterectomy by achieving equal or inferior periprocedural risk. ${ }^{4,21,22}$ However, a subgroup analysis of data of some recent trials failed to demonstrate a statistically significant difference between protected and unprotected CAS. It has been argued that the relative reduction provided by CPDs may be as low as 25\%, probably because the devices themselves serve as sources for emboli during the procedure or removal technique. ${ }^{23}$ In general, 30-day adverse outcome (stroke/death rate) for CAS with the use of CPDs ranged from $1.2 \%$ to $7 \%$, whereas for CAS without protection, it ranged from $2.4 \%$ to $11 \% .^{20}$

Thus, there continues to be controversy regarding the benefit of the CPDs.

On the basis of our experience, the use of CPDs is not required to treat carotid stenoses because balloon angioplasty is not performed either before or after stent placement. It is well-known that the highest potential for embolization occurs post-stent placement when the balloon crushes the plaque against the stent struts. ${ }^{24,25}$

In our series, the 30-day composite rate of any stroke or death was $1.2 \%$ ( $0.8 \%$ for symptomatic and $0.4 \%$ for asymptomatic patients). These results are considerably below the $3 \%$ and $6 \%$ of maximum complication rates recommended in the American Heart Association guidelines for carotid endarterectomy of asymptomatic and symptomatic patients, respectively, ${ }^{17}$ and compare favorably with rates found in patients enrolled in large trials of carotid stent placement $(10 \%$ in the Carotid and Vertebral Artery Transluminal Angioplasty Study, ${ }^{26} 12.1 \%$ in the WALLSTENT [carotid stenting versus endarterectomy in patients with symptomatic carotid stenosis] trial, ${ }^{27} 4.8 \%$ in the Stenting and Angioplasty with Protection in Patients at High Risk for Endarterectomy study, ${ }^{4} 6.8 \%$ in the Stent-Protected Angioplasty versus Carotid Endarterectomy trial, ${ }^{2}$ and $9.6 \%$ in the EVA-3S [endarterectomy versus stenting in patients with symptomatic severe carotid stenosis] study $^{1}$ ).

Unlike peripheral or coronary stenoses, carotid stenoses are very infrequently symptomatic due to hemodynamic compromise. Rather, symptoms arise from embolization from a carotid plaque. Therefore, it remains to be determined what degree of correction of carotid stenosis is necessary to reduce the risk of embolization, because on one hand, it is known that balloon dilation to achieve total expansion at once might produce a higher risk of procedural complications such as additional emboli; on the other hand, leaving residual stenosis may lead to a higher rate of late restenosis, which at this time is of uncertain clinical significance. ${ }^{28}$ Some CAS trials have defined technical success as residual stenosis of $<30 \%$. ${ }^{4}$ Others have used a definition of residual stenosis of $<50 \%{ }^{29}$ In the absence of definitive scientific evidence, technical success was arbitrarily defined as stent placement resulting in improvement of the stenosis by $\geq 20 \%$, with a final residual stenosis of $<50 \%$ with NASCET measurement criteria. ${ }^{28}$

In our series, the degree of stenosis decreased from a mean of $82 \%$ before the procedure to a mean of $30 \%$ immediately after stent placement. In 156 patients, the immediate residual stenosis was $<30 \%$; in 79 , between $30 \%$ and $50 \%$; and in 20 patients, $>50 \%$ (in all these patients, there was at least a $20 \%$ improvement of stenosis).

The rate of in-stent restenosis appears to be higher when carotid stenoses are treated with stents alone. Bussière et $\mathrm{al}^{30}$ reported a restenosis rate of $15.9 \%$ at 1 year by using this technique. In our series, restenosis occurred in 19 (7.4\%) pa- 

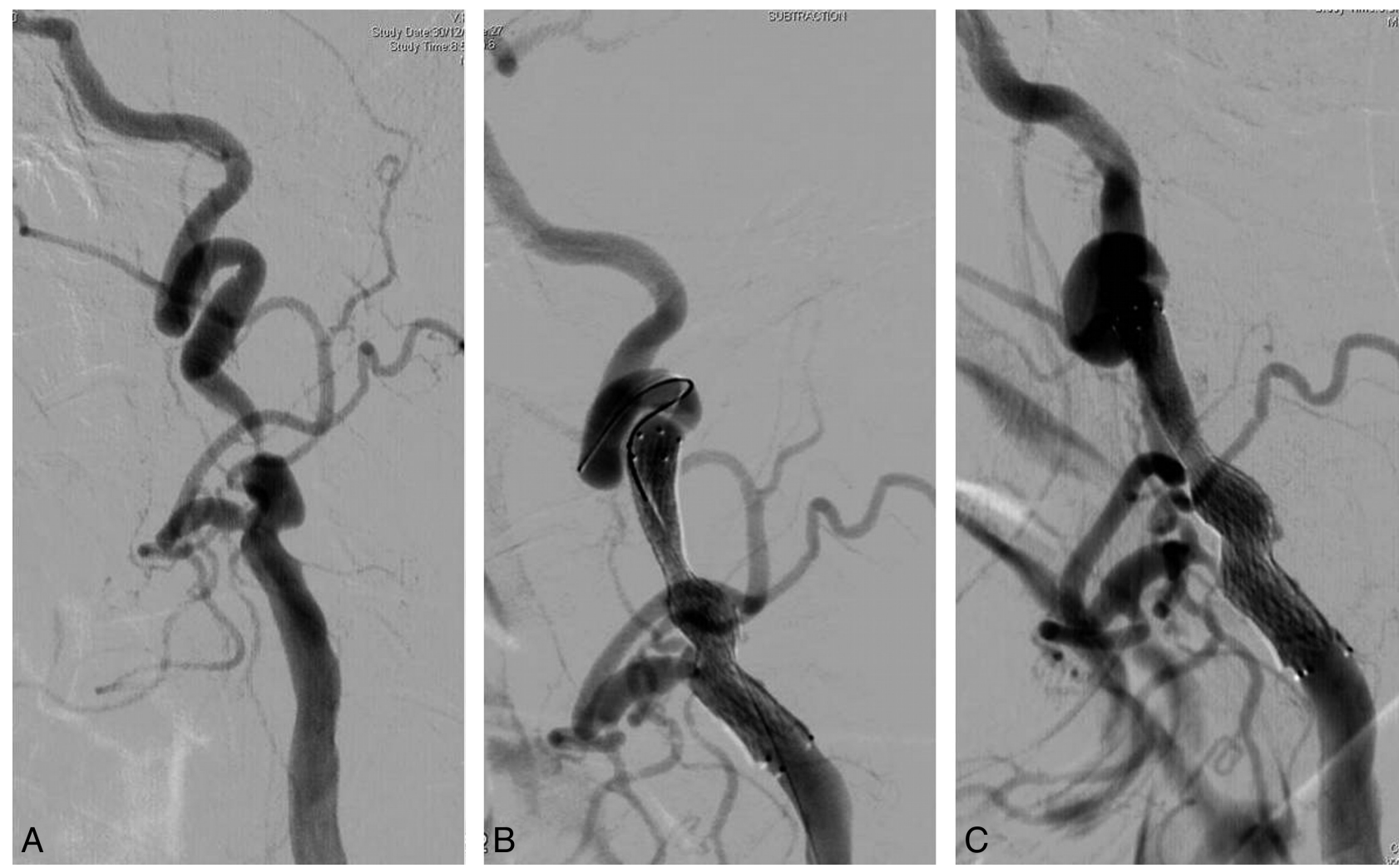

Fig 1. Severe CAS. The tortuosity of the poststenotic segment of the internal carotid artery might have precluded filter placement. Without the use of CPDs, the stent could be placed easily, obtaining a $30 \%$ residual stenosis in the immediate angiographic control image.

tients; lack of stent expansion, in 14 (5.4\%); and a combination of both, in 5 (1.9\%). Fortunately, most in-stent restenoses are asymptomatic, as evidenced by the low rates of ipsilateral stroke during follow-up. Strokes arising from carotid stenosis are most often due to atheroembolism. Treatment, therefore, should be aimed at eliminating the potential for plaque embolization. Restoration of a normal lumen diameter at once should be considered a secondary goal. Although the criteria for re-intervention are not well-defined, angioplasty with protection was performed in all of these otherwise asymptomatic patients.

Carotid sinus reaction including bradycardia, asystole, and hypotension is 1 of the most common complications of internal carotid artery angioplasty. It may occur due to an abnormal response to a baroreflex related to overstretching of the carotid sinus caused by the dilating balloon. In the published literature, the rate of hypotension after CAS varies from $10 \%$ to $42 \%$, and the rate of bradycardia, from $27 \%$ to $37 \% .{ }^{31}$ The sustained outward force of a self-expanding stent seems to be a weak stimulus to the baroreceptors of the carotid sinus, whereas forceful dilation with a balloon is a strong stimulus; therefore, these adverse events can be drastically reduced if neither prestenting nor poststenting balloon dilation is used. Hypotension and bradycardia, in our series, occurred in 5.1\% of the cases during the procedure; none required vasopressor drugs or a stay in the ICU for hemodynamic monitoring.

An additional benefit of the gradual expansion of the stent with time might be the reduction of the risk of reperfusion syndrome after restoration of carotid flow. Although hyperperfusion did occur in 4 patients, hemorrhage was not de- tected in any on CT. This hypothesis, nevertheless, can only be confirmed in large randomized trials comparing traditional protected CAS with balloon angioplasty and CASWBP.

DWI is currently the most sensitive tool for detecting early cerebral ischemia and offers the possibility of revealing small and thus asymptomatic lesions occurring during CAS, so it would have considerable use in evaluating the efficacy of CPDs. In 82 patients, MR imaging with DWI could be performed before and within 48 hours after the procedure, depicting $8.5 \%$ of silent infarcts. This result compares favorably with previous reports that reveal a number of silent ischemic lesions after neuroprotected CAS, ranging from $15.3 \%$ to $50 \% .^{32,33}$ This finding supports the idea that less device manipulation, including CPDs, across the lesion minimized emboli dislodgment.

In these patients, only 2 types of stents were used, and both have an open-cell design. All TIAs in this series occurred several hours after the procedure, so it is possible that the use of different stents, particularly those with closed-cell design in echolucent lesions, could decrease neurologic complication rates by covering a greater percentage of the vascular wall within the stented region and avoiding dislodgment of the plaque while the stent expands.

Finally, with this technique, in which a CPD is not needed because angioplasty is not performed, indications for CAS can be extended. There are some anatomic difficulties such as tortuous vessels, in which CPDs are not suitable for navigation or placement; in these cases, our technique may overcome such difficulties, making the procedure possible and safer (Fig 1). 


\section{Conclusions}

On the basis of our experience, stent placement alone might be enough to treat patients who have symptomatic or asymptomatic severe carotid stenosis. In-stent restenosis rates with this approach seem to be higher than those with conventional angioplasty and stent placement. However, angioplasty may be performed safely a second time if necessary.

CASWBAP may reduce the occurrence of hemodynamic depression during and after the procedure, avoiding the need for vasopressor support and the ICU, thus reducing in-hospital stays and costs. Further clinical investigations, comparing CASWBAP directly with the protected CAS with balloon angioplasty, deserve the consideration of the scientific community.

\section{Acknowledgments}

We thank Juan Manuel Ramos Goñi for statistical advice.

\section{References}

1. Mas JL, Chatelier G, Beyssen B, et al, for the EVA-3S Investigators. Endarterectomy versus stenting in patients with symptomatic severe carotid stenosis. N Engl J Med 2006;355:1660-71

2. Ringleb PA, Allenberg J, Bruckmann $H$, et al. $\mathbf{3 0}$ day results from the SPACE trial of stent-protected angioplasty versus carotid endarterectomy in symptomatic patients: a randomized non-inferiority trial. Lancet 2006;368:123947. Erratum in: Lancet 2006;368:1238

3. Ederle J, Dobson J, Featherstone RL, et al, for the International Carotid Stenting investigators. Carotid artery stenting compared with endarterectomy in patients with symptomatic carotid stenosis (International Carotid Stenting Study): an interim analysis of a randomised controlled trial. Lancet 2010;375: 985-97. Epub 2010 Feb 25. Erratum in: Lancet 2010;376:90

4. Yadav JS, Wholey MH, Kuntz RE, et al, for the Stenting and Angioplasty with Protection in Patients at High Risk for Endarterectomy Investigators. Protected carotid artery stenting versus endarterectomy in high-risk patients. $N$ Engl J Med 2004;351:1493-501

5. Cambria RP. Stenting for carotid-artery stenosis. N Engl J Med 2004;351: $1565-67$

6. Steinbauer MG, Pfister K, Greindl M, et al. Alert for increased long-term follow-up after carotid artery stenting: results of a prospective, randomized, single-center trial of carotid artery stenting vs carotid endarterectomy. $J$ Vasc Surg 2008;48:93-98

7. Kastrup A, Groschel K, Krapf H, et al. Early outcome of carotid angioplasty and stenting with and without cerebral protection devices: a systematic review of the literature. Stroke 2003;34:813-19

8. Castriota F, Cremonesi A, Manetti R, et al. Impact of cerebral protection devices on early outcome of carotid stenting. J Endovasc Ther 2002;9:786-92

9. Cardaioli P, Giordan M, Panfili M, et al. Complication with an embolic protection device during carotid angioplasty. Catheter Cardiovasc Interv 2004;62:234-36

10. Higashida RT, Meyers PM, Phatouros CC, et al. Reporting standards for carotid artery angioplasty and stent placement. J Vasc Interv Radiol 2004; $15: 421-22$

11. Coggia M, Goeau-Brissonniere O, Duval JL, et al. Embolic risk of the different stages of carotid bifurcation balloon angioplasty: an experimental study. $J$ Vasc Surg 2000;31:550-57
12. Maynar M, Baldi S, Rostagno R, et al. Carotid stenting without use of balloon angioplasty and distal protection devices: preliminary experience in 100 cases. AJNR Am J Neuroradiol 2007;28:1378-83

13. Mathiesen EB, Bonaa KH, Joakimsen O. Low levels of high-density lipoprotein cholesterol are associated with echolucent carotid artery plaques: the TROMSO study. Stroke 2001;32:1960-65

14. Gronholdt ML, Nordestgaard BG, Schroeder TV, et al. Ultrasonic echolucent carotid plaques predict future strokes. Circulation 2001;104:68-73

15. Geroulakos G, Hobson RW, Nicolaides A. Ultrasonographic carotid plaque morphology in predicting stroke risk. Br J Surg 1996;83:582-87

16. North American Symptomatic Carotid Endarterectomy Trial Collaborators. Beneficial effect of carotid endarterectomy in symptomatic patients with high-grade carotid stenosis. N Engl J Med 1991; 325;445-53

17. Moore WS, Barnett HJ, Beebe HG, et al. Guidelines for carotid endarterectomy: a multidisciplinary consensus statement from the ad hoc committee, American Heart Association. Stroke 1995;26:188-201

18. Roffi M, Yadav JS. Carotid stenting. Circulation 2006;114:e1-4

19. Roubin GS, Iyer S, Halkin A, et al. Realizing the potential of carotid artery stenting: proposed paradigms for patient selection and procedural technique. Circulation 2006;113:2021-30

20. Goodney PP, Schermerhorn ML, Powell RJ. Current status of carotid artery stenting. J Vasc Surg 2006;43:406-11

21. CARESS Steering Committee. Carotid revascularization using endarterectomy or stenting systems (CARESS): phase I clinical trial. J Endovasc Ther 2003;10:1021-30

22. Brott TG, Hobson RW 2nd, Howard G, et al, for the CREST Investigators. Stenting versus endarterectomy for treatment of carotid-artery stenosis. N Engl J Med 2010;363:11-23

23. Cloft HJ. Distal protection: maybe less than you think. AJNR Am J Neuroradiol 2008;29:407-08

24. Manninen HI, Rasanen HT, Vanninen RL, et al. Stent placement versus percutaneous transluminal angioplasty in cadavers in situ: distal embolization and findings at intravascular US-MR imaging and histopathologic analysis. $R a$ diology 1992;212:483-92

25. Vos JA, Van den Berg JC, Ernst SM, et al. Carotid angioplasty and stent placement: comparison transcranial Doppler US data and clinical outcome with and without filtering cerebral protection devices in 509 patients. Radiology 2005;234:493-99

26. Endovascular versus surgical treatment in patients with carotid stenosis in the Carotid and Vertebral Artery Transluminal Angioplasty Study (CAVATAS): a randomized trial. Lancet 2001;357:1729-37

27. Alberts MJ. Results of a multicenter prospective randomized trial of carotid artery stenting vs. carotid endarterectomy. Stroke 2001;32:325

28. Barr JD, Connors JJ 3rd, Sacks D, et al. Quality improvement guidelines for the performance of cervical carotid angioplasty and stent placement. J Vasc Interv Radiol 2003;14(9 pt 2):S321-35

29. Gray WA, Hopkins LN, Yadav S, et al, for the ARCHeR Trial Collaborators. Protected carotid stenting in high-surgical-risk patients: the ARCHeR results. J Vasc Surg 2006;44:258-68

30. Bussière M, Pelz DM, Kalapos $\mathrm{P}$, et al. Results using a self-expanding stent alone in the treatment of severe symptomatic carotid bifurcation stenosis. J Neurosurg 2008;109:454-60

31. Lavoie P, Rutledge J, Dawoud MA, et al. Predictors and timing of hypotension and bradycardia after carotid artery stenting. AJNR Am J Neuroradiol 2008;29:1942-47

32. Palombo G, Faraglia V, Stella N, et al. Late evaluation of silent cerebral ischemia detected by diffusion-weighted MR imaging after filter-protected carotid artery stenting. AJNR Am J Neuroradiol 2008;29:1340-43

33. Bonati L, Jongen L, Haller S, et al. New ischemic brain lesions on MRI after stenting or endarterectomy for symptomatic carotid stenosis: a substudy of the International Carotid Stenting Study (ICSS). Lancet Neurol 2010;9: $353-62$ 